\title{
METHANOL AND $\mathrm{H}_{2} \mathrm{O}$ MASERS IN A DISK AROUND GL 2789
}

\author{
V. I. Slysh ${ }^{1}$, M. A. Voronkov ${ }^{1}$, I. E. Val'tts ${ }^{1}$, \\ V. Migenes $^{2}$ \\ 1 - Astro Space Center, Lebedev Physical Institute, 84/32 Prof- \\ soyuznaya ul., 117997 Moscow, Russia \\ 2 - Departamento de Astronomía, Universidad de Guanjuato, \\ Guanjuato, Mexico
}

Received on March 20, 2002; In final form on May 23, 2002

\begin{abstract}
VLBA and EVN radio observations of $\mathrm{H}_{2} \mathrm{O}$ masers at $22 \mathrm{GHz}$ and methanol masers at $6.7 \mathrm{GHz}$ have been used to obtain images of the maser spots in the infrared object GL 2789, which is associated with the young stellar object V645 Cyg. The position of these masers coincides with that of the optical object to within 0 "'.2. The maser spots are located in a line oriented north-south, and their positions and radial velocities can be described by a model with a Keplerian disk with maximum radius $40 \mathrm{AU}$ for the $\mathrm{H}_{2} \mathrm{O}$ masers and $800 \mathrm{AU}$ for the methanol masers. The $\mathrm{H}_{2} \mathrm{O}$ and methanol masers spots are unresolved, and the lower limits for their brightness temperatures are $2 \times 10^{13} \mathrm{~K}$ and $1.4 \times 10^{9} \mathrm{~K}$, respectively. A model in which the maser radiation is formed in extended water-methanol clouds associated with ice planets forming around the young star is proposed.
\end{abstract}

\section{INTRODUCTION}

GL 2789 is an infrared source that coincides with an optical reflection nebula and the star-like object V645 Cyg [1]. The optical image contains the star-like condensation N0 and several filamentary nebulae, the brightest of which is called N1. Cohen [1] suggests that this object is associated with a young $\mathrm{O} 7 \mathrm{star}$ at a distance of $6 \mathrm{kpc}$ that is surrounded by a bipolar nebula. Humphreys et al. [2] concluded that the optical spectrum of the nebulosity around V645 Cyg corresponded to that of an A star surrounded by a 
shell, and estimated a smaller distance to the object of about $3.5 \mathrm{kpc}$ (see also Goodrich [3]). In both cases, the mass of the star was estimated to be $10 M_{\odot}$. V645 Cyg is located at the center of a molecular cloud that emits $\mathrm{CO}$ and $\mathrm{NH}_{3}$ lines, with the $\mathrm{CO}$ line emission showing signs of a bipolar outflow $[4,5]$. The optical object V645 Cyg is also located at the center of a source of thermal radio continuum emission about $7^{\prime \prime}$ in size [6]. Lada et al. [7] discovered $\mathrm{H}_{2} \mathrm{O}$ masers with radial velocities of $-48.9 \mathrm{~km} / \mathrm{s}$ and $-44.5 \mathrm{~km} / \mathrm{s}$, whose position was determined with an accuracy of 0.2 and coincides with the position of the optical component N0. The VLA observations of Tofani et al. [8] obtained 13 years later showed $\mathrm{H}_{2} \mathrm{O}$ masers with radial velocities of $-43.3 \mathrm{~km} / \mathrm{s}$ and $-41.0 \mathrm{~km} / \mathrm{s}$ at the same position. $\mathrm{OH}$ maser emission at $1665 \mathrm{MHz}$ at radial velocities ranging from $-45 \mathrm{~km} / \mathrm{s}$ to $-41.6 \mathrm{~km} / \mathrm{s}$ was detected by Morris and Kazés [9]. Slysh et al. [10] detected a class II maser in the $5_{1}-6_{0} A^{+}$methanol line at $6.7 \mathrm{GHz}$ at radial velocities from $-43.5 \mathrm{~km} / \mathrm{s}$ to $-40.5 \mathrm{~km} / \mathrm{s}$. Since maser emission is associated with early stages of stellar evolution, the presence of $\mathrm{OH}, \mathrm{H}_{2} \mathrm{O}$, and methonol masers suggests that V645 Cyg is a protostar or young stellar object. Investigations of the fine structure of the maser sources could shed light on mechanisms for interactions between the young star or protostar and its surrounding medium. Here, we present the results of high-angular-resolution (milliarcsecond-scale) studies of the structure of the $\mathrm{H}_{2} \mathrm{O}$ and methanol masers associated with this source.

\section{OBSERVATIONS}

The $\mathrm{H}_{2} \mathrm{O}$-maser observations of GL 2789 were carried out at $22 \mathrm{GHz}$ on the VLBA on June 5, 1996 as part of a prelaunch survey for the VSOP spaceVLBI project [11]. The VLBA consists of ten 25-m radio telescopes located at various points on US territory, and provides a maximum baseline length of about $8000 \mathrm{~km}$. The observations were conducted in a snapshot mode with the individual scans being five minutes in duration. The resulting synthesized beam was $0.3 \times 0.95$ mas. The total recorded bandwidth of $8 \mathrm{MHz}$ was divided into 512 spectral channels during the correlation of the data, providing a spectral resolution of $15.6 \mathrm{kHz}$ per channel or $0.21 \mathrm{~km} / \mathrm{s}$. The sensitivity achieved during the five-minute accumulation time was $100 \mathrm{mJy} / \mathrm{beam}$. The delay and bandpass calibration was performed using observations of the continuum sources 3C 273 and 3C 84. The post-correlation reduction included 


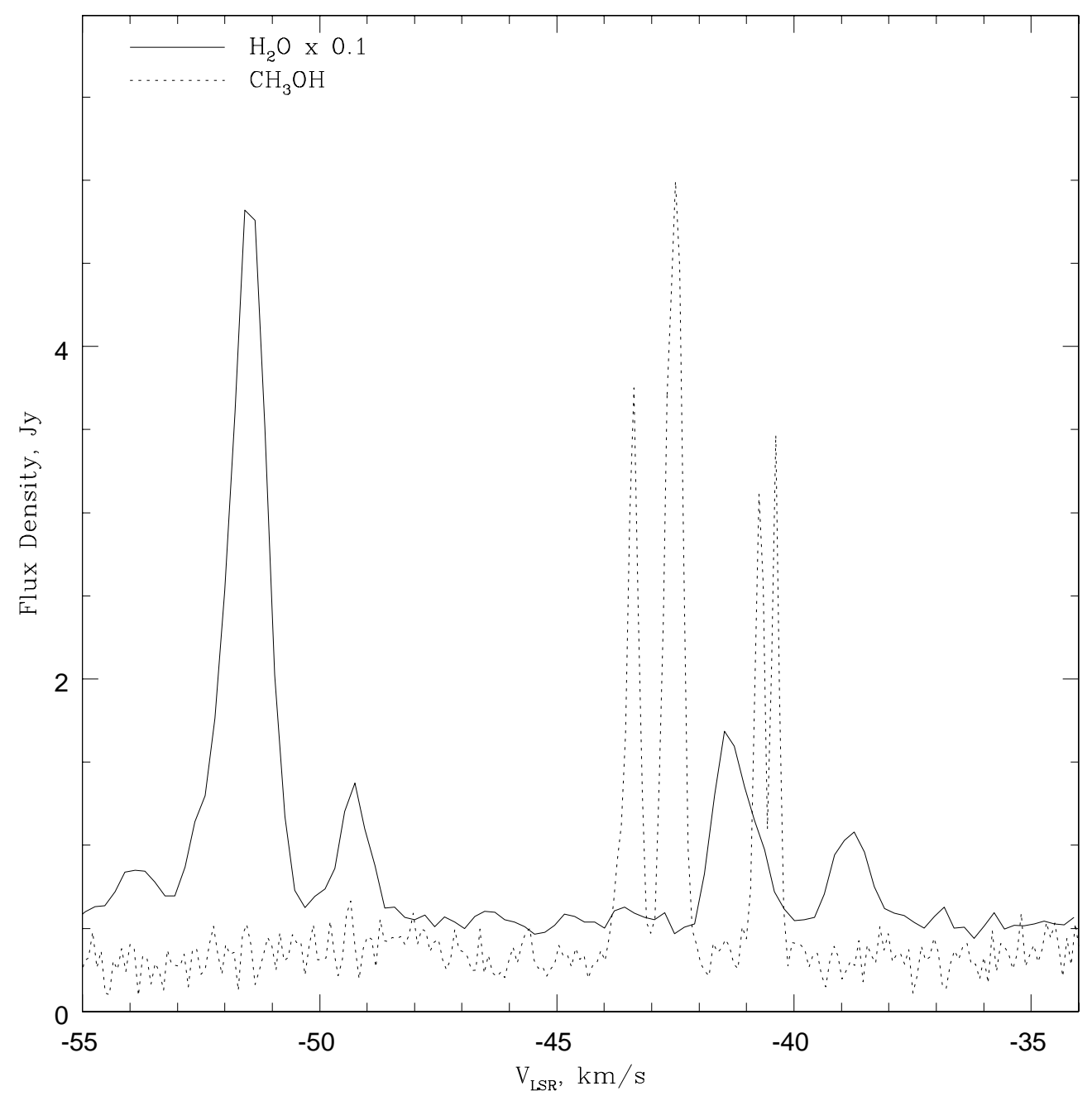

Figure 1: Water vapor (solid) and methanol (dotted) maser spectra of GL 2789. The $\mathrm{CH}_{3} \mathrm{OH}$ maser spectrum was constructed from observations obtained in 1998. The flux density of the $\mathrm{H}_{2} \mathrm{O}$ maser has been decreased by a factor of ten.

amplitude calibration and fringe fitting. The fringe fitting was carried out in two stages: (1) the residual delays for the radio telescopes were determined using the continuum sources and (2) the residual fringe rate was determined for each of the sources individually using its strongest spectral features. We also applied a correction for the Doppler shift of each telescope. 
Observations of the class II methonol maser in the $5_{1}-6_{0} A^{+}$transition were carried out at $6.7 \mathrm{GHz}$ on the European VLBI network (EVN) in 1998 and 2000. Five telescopes equiped with $6.7-\mathrm{GHz}$ receivers participated: the $100 \mathrm{~m}$ Effelsberg, $25 \mathrm{~m}$ Jodrell Bank, $32 \mathrm{~m}$ Medicina (Noto in 2000), $25 \mathrm{~m}$ Onsala, and $32 \mathrm{~m}$ Torun antennas. In 1998 and 2000, GL 2789 was observed during four and three 10-min scans, respectively. The resulting synthesized beams were $4.3 \times 8.2$ mas. The recorded bandwidth of $2 \mathrm{MHz}$ was divided into 1024 spectral channels, providing a spectral resolution of $1.95 \mathrm{kHz}$ or $0.088 \mathrm{~km} / \mathrm{s}$. The sensitivity of the observations was $400 \mathrm{mJy} /$ beam. The delay and bandpass calibration was conducted using observations of several continuum sources. The post-correlation reduction of the methanol observations was carried out in the same way as the reduction of the $\mathrm{H}_{2} \mathrm{O}$-maser data.

The subsequent processing of the data consisted of determining the absolute coordinates of the masers and the relative positions of the maser spots, and constructing images of the spots themselves. This was carried out in the AIPS package.

\section{RESULTS}

\subsection{Spectra}

Figure 1 presents the spectra of the $\mathrm{H}_{2} \mathrm{O}$ (solid) and methonol (dotted) masers observed in 1998. Each spectrum contains four features, however the $\mathrm{H}_{2} \mathrm{O}$ maser occupies a broader velocity interval than the methanol maser. The entire methanol spectrum is located in the red half of the $\mathrm{H}_{2} \mathrm{O}$ radialvelocity spectrum. No methanol maser feature coincides in radial velocity with any of the $\mathrm{H}_{2} \mathrm{O}$ maser features. A comparison with the earlier observations of Lada et al. [7] and Tofani et al. [8] shows that the $\mathrm{H}_{2} \mathrm{O}$ spectra experienced substantial variations. In 1998, the brightest feature had a radial velocity of $-51.5 \mathrm{~km} / \mathrm{s}$, while the brightest features in 1979 [7] and 1992 [8] had radial velocities of $-50.2 \mathrm{~km} / \mathrm{s}$ and $-43.3 \mathrm{~km} / \mathrm{s}$, respectively. Features at radial velocities of $-39 \mathrm{~km} / \mathrm{s}$ and $-41.5 \mathrm{~km} / \mathrm{s}$ have also appeared. The methanol maser spectrum for the 1998 EVN observations differs markedly from a spectrum observed at Medicina in 1995, but nearly coincides with the spectrum obtained by Szymczak et al. [12] in 1999. While the brightest feature in 1995 had a radial velocity of $-43.6 \mathrm{~km} / \mathrm{s}$ and a flux of $19 \mathrm{Jy}$, 


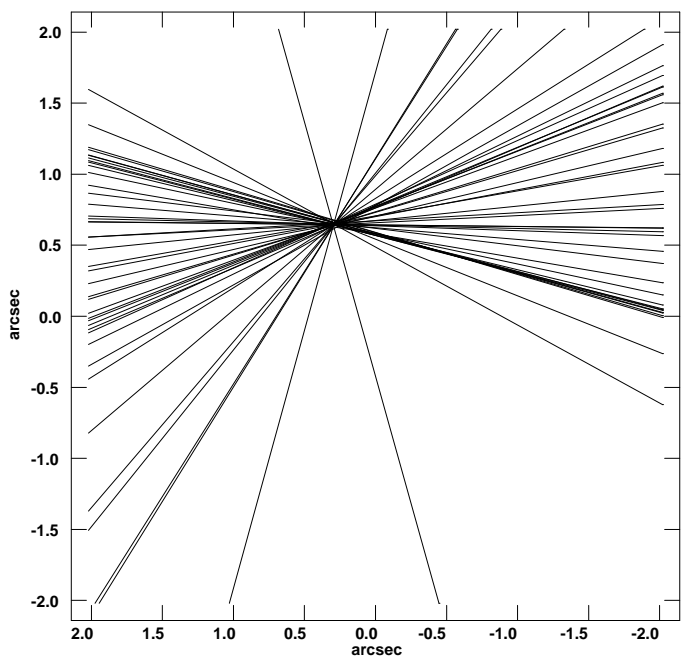

(a)

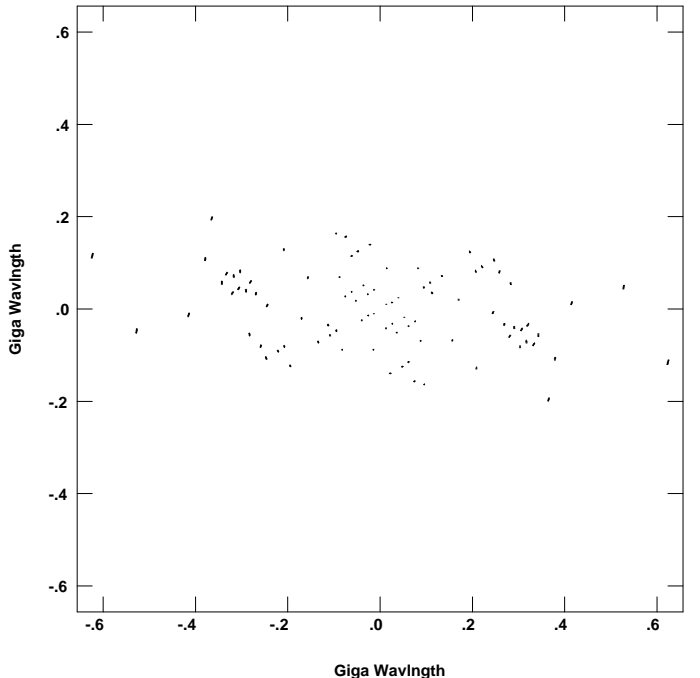

(b)

Figure 2: (a) Determination of the absolute coordinates of the $\mathrm{H}_{2} \mathrm{O}$ maser $(v=-51.8 \mathrm{~km} / \mathrm{s})$. The shift of the intersection point from the phase center $(0,0)$ is $\Delta \alpha=293.9 \pm 11.6$ mas, $\Delta \delta=650.8 \pm 3.7$ mas, and corresponds to the difference between the true maser coordinates at epoch and the coordinates used during correlation. (b) $u v$ coverage for the observations of the $\mathrm{H}_{2} \mathrm{O}$ maser in GL 2789.

the brightest features in 1998, 1999, and 2000 were at radial velocities of $-42.5 \mathrm{~km} / \mathrm{s}$ (flux $7.5 \mathrm{Jy}$ ), $-40.9 \mathrm{~km} / \mathrm{s}$ (flux $7 \mathrm{Jy}$ ), and $-40.4 \mathrm{~km} / \mathrm{s}$ (flux $27 \mathrm{Jy})$, respectively. At the same time, the character of the spectrum remained nearly unchanged, always containing the same four features, with only their relative fluxes varying.

\subsection{Absolute coordinates}

We determined the absolute coordinates of the masers using the method of fringe-rate mapping. Figure $2 \mathrm{a}$ shows the result of measuring the absolute coordinates of the $\mathrm{H}_{2} \mathrm{O}$ feature at $-51.8 \mathrm{~km} / \mathrm{s}$. Each line corresponds to the locus of possible source positions for which the fringe rate for one of the VLBA baselines is equal to the measured value. The number of lines is equal to the number of baselines (the number of pairs of antennas). Since the VLBA consists of ten antennas, the number of baselines is 45 . The point 
Table 1: Coordinates of the brightest $\mathrm{H}_{2} \mathrm{O}$ and $\mathrm{CH}_{3} \mathrm{OH}$ maser features in GL 2789

\begin{tabular}{|c|c|c|c|}
\hline Measurement & $\alpha_{2000}$ & $\Delta_{2000}$ & Reference \\
\hline Optical, N0 & $21^{h} 39^{m} 58.27 \pm 0.03$ & $50^{\circ} 14^{\prime} 20^{\prime \prime} .9 \pm 0^{\prime \prime} .2$ & Cohen [1] \\
\hline Radio, 3.6 and $6 \mathrm{~cm}$, VLA & $2139 \quad 58.26 \pm 0.05$ & $\begin{array}{lll}50 & 14 & 21.3 \pm 0.5\end{array}$ & Skinner et al. [6] \\
\hline $\mathrm{H}_{2} \mathrm{O}$ maser, VLA $(-43.3 \mathrm{~km} / \mathrm{s})$ & $58.27 \pm 0.01$ & $50 \quad 14 \quad 21.0 \pm 0.1$ & Tofani et al. [8] \\
\hline $\mathrm{H}_{2} \mathrm{O}$ maser, VLBI $(-50.2 \mathrm{~km} / \mathrm{s})$ & $58.28 \pm 0.03$ & $1421.0 \pm 0.2$ & Lada et al. [7] \\
\hline $\mathrm{H}_{2} \mathrm{O}$ maser, VLBA $(-51.8 \mathrm{~km} / \mathrm{s})$ & $58.277 \pm 0.001$ & $1421.041 \pm 0.005$ & Current paper \\
\hline Methanol, EVN $1998(-42.5 \mathrm{~km} / \mathrm{s})$ & $58.286 \pm 0.006$ & $20.98 \pm 0.05$ & Current paper \\
\hline Methanol, EVN $2000(-42.5 \mathrm{~km} / \mathrm{s})$ & $58.286 \pm 0.005$ & $20.6 \pm 0.2$ & Current paper \\
\hline
\end{tabular}

of intersection of all the lines corresponds to the true position of the source. The accuracy of the absolute coordinates of $\mathrm{H}_{2} \mathrm{O}$ masers obtained in this way varies from fractions of an arcsecond to several milliarcseconds. The coordinates of the methanol masers were determined in the same fashion.

Table 1 presents the coordinates of the brightest maser features. The table presents the coordinates obtained from both our 1998 and 2000 methanol observations, which agree within their errors $(<2 \sigma)$. The coordinates of the $\mathrm{H}_{2} \mathrm{O}$ maser coincide with those presented by Lada et al. [7] and Tofani et al. [8] within their errors, although they correspond to different spectral features. The accuracy for the $\mathrm{H}_{2} \mathrm{O}$ maser coordinates provided by our observations was more than an order of magnitude higher than for the observations of Lada et al. [7] and Tofani et al. [8]. The methanol maser is shifted relative to the $\mathrm{H}_{2} \mathrm{O}$ maser by $0^{\prime \prime} .08 \pm 0^{\prime \prime} .07$ to the east and $0^{\prime \prime} .09 \pm 0^{\prime \prime} .05$ to the south. The coordinates of both masers coincide with those for the optical object N0 and the source of continuum radio emission, which are known with much lower accuracy. Nevertheless, we can assert with certainty that the masers are located within the extended continuum radio source, which has a diameter of $7^{\prime \prime}$.

\subsection{The Images}

\subsubsection{The $\mathrm{H}_{2} \mathrm{O}$ maser}

To construct the image of the $\mathrm{H}_{2} \mathrm{O}$ maser, we performed self-calibration of the VLBA data using the strongest spectral feature, at a radial velocity of $-51.8 \mathrm{~km} / \mathrm{s}$. We were not able to calibrate using external sources, i.e., strong point-like continuum sources, since the amplitude and phase characteristics of the array did not remain sufficiently constant between observations of 
the calibrator and maser sources, due to changing conditions in the atmosphere. During the self-calibration on a maser feature, the amplitude and phase characteristics of the antennas were determined simultaneously with the image of the maser itself, and variations in these characteristics and in the atmospheric characteristics were removed from the data.

Figure $2 \mathrm{~b}$ shows the coverage of the $u v$ plane for the observations of GL 2789. Since each of the individual observations was short (5 min), the tracks corresponding to individual pairs of antennas are represented by short segments of ellipses that appear nearly like dots. We can see that the range for $u$ (horizontal direction) is appreciably larger than the range for $v$ (vertical direction). This is due to the locations of the antennas in the VLBA, whose extent in the east-west direction is somewhat larger than in the north-south direction. As a result, the synthesized beam has an elliptical shape with a $3: 1$ axial ratio.

The self-calibration procedure yielded an image of the reference feature at $-51.8 \mathrm{~km} / \mathrm{s}$, as well as images of all the remaining features with radial velocities from $-38.0 \mathrm{~km} / \mathrm{s}$ to $-55 \mathrm{~km} / \mathrm{s}$. The maser spots are concentrated primarily in two groups. The southern group contains features with radial velocities from $-49 \mathrm{~km} / \mathrm{s}$ to $-55 \mathrm{~km} / \mathrm{s}$, while radial velocities in the northern group are from $-38 \mathrm{~km} / \mathrm{s}$ to $-42 \mathrm{~km} / \mathrm{s}$. Figures 3 and 4 present images of the maser spots of the northern and southern groups in all channels where there was an appreciable signal. These images are super-resolved; i.e., the images were convolved with a beam 0.1 mas in size. This is admissible in the presence of high signal to noise. A map of the masers in the southern group constructed for all the channels is shown in Fig. 5a, an analogous map for the northern group is shown in Fig. 5b, and a combined map is shown in Fig. 5c. We can see that the southern group of maser spots forms a structure that is elongated in the east-west direction, while the northern group is elongated roughly north-south. Within the northern group, the radial velocity decreases from south to north, contrary to the general tendency for the radial velocity for the maser as a whole to grow from south to north. One weak feature with a radial velocity of $-50.5 \mathrm{~km} / \mathrm{s}$ has a peculiar location, separated from the southern group by about 5.6 mas to the southwest. In all, we detected eight maser spots in the image of GL 2789. Their mutual locations relative to the reference feature at $-51.8 \mathrm{~km} / \mathrm{s}$ are presented in Table 2 and shown in Fig. 5d. This maser source was the most compact of all those observed during the survey: the maximum distance between individual features does not exceed 10 mas. 


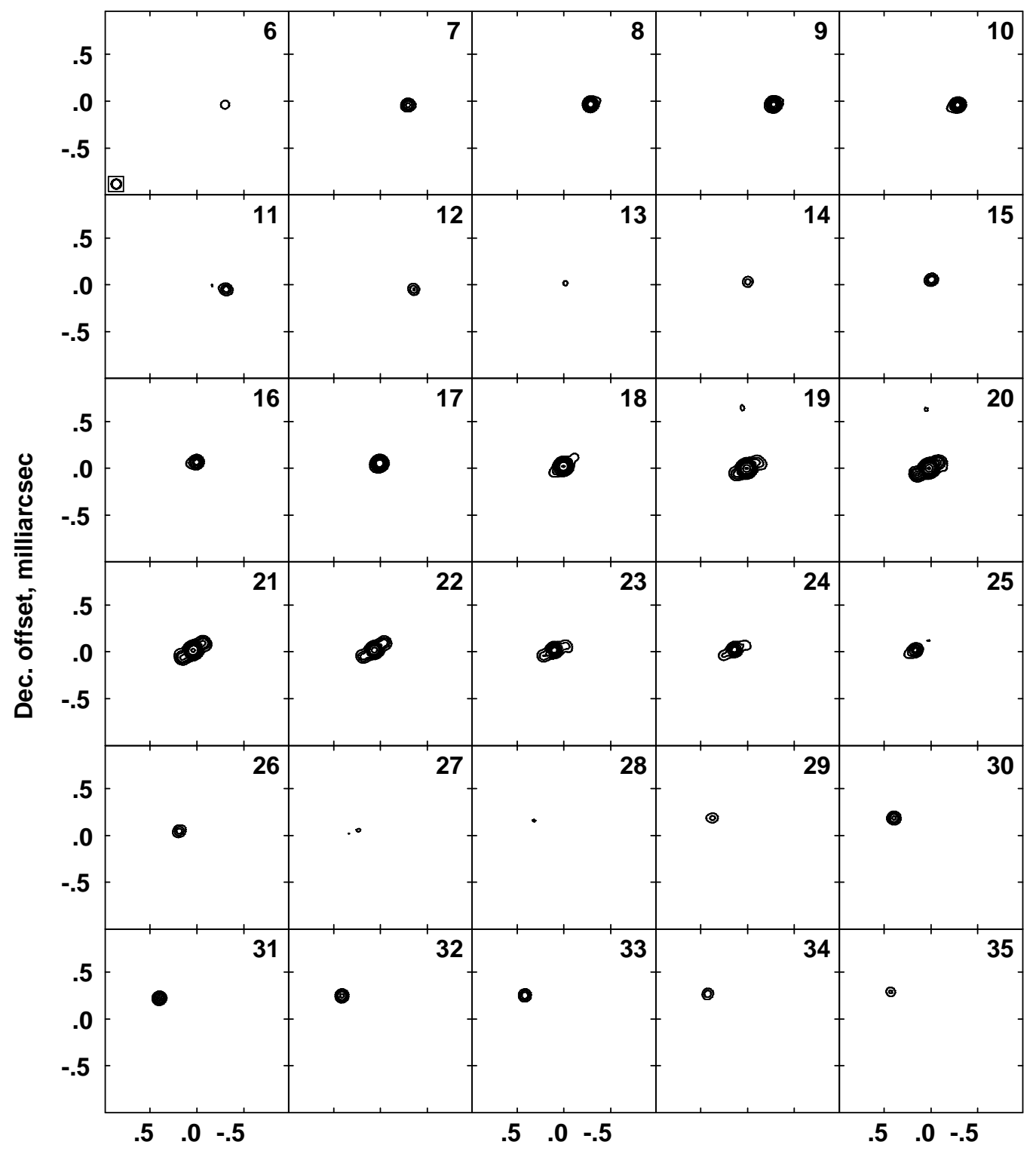

R.A. offset, milliarcsec

Figure 3: Images of the southern group of water maser spots in various channels. The contours are drawn at $3.7 \times$ $(0.3,0.5,0.7,1,1.2,1.5,2,3,5,6,7,8,9) \mathrm{Jy} /$ beam. 


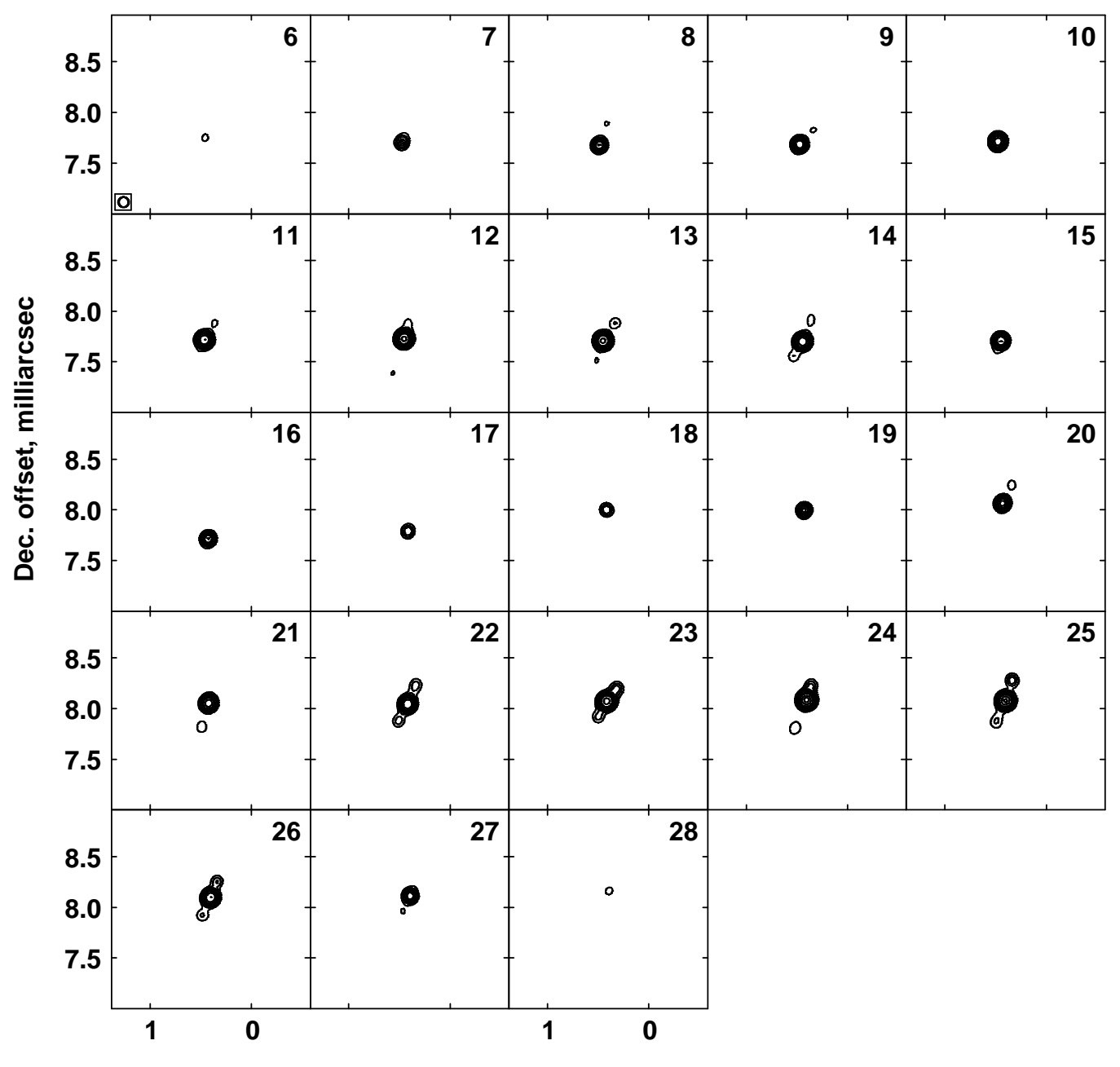

R.A. offset, milliarcsec

Figure 4: Images of the northern group of water maser spots in various channels. The contours are drawn at $1.1 \times$ $(0.3,0.5,0.7,1,1.2,1.5,2,3,5,6,7,8,9) \mathrm{Jy} /$ beam. 


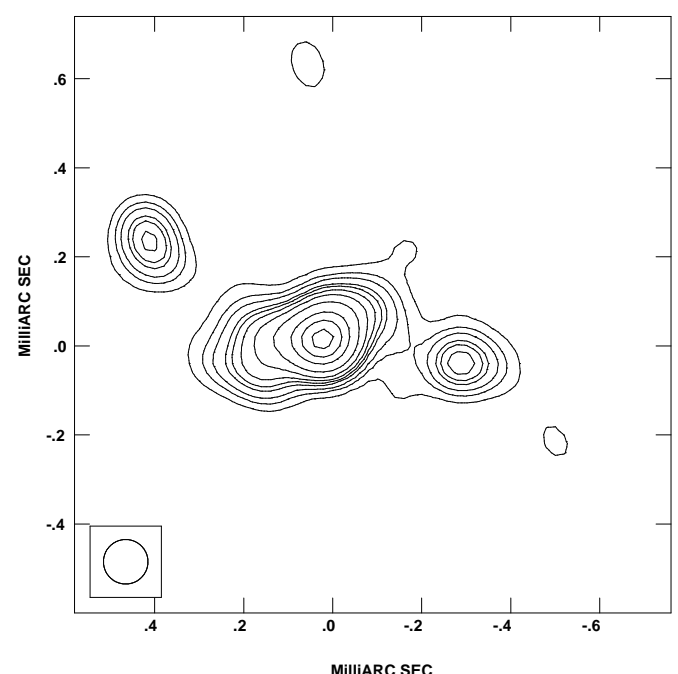

(a)

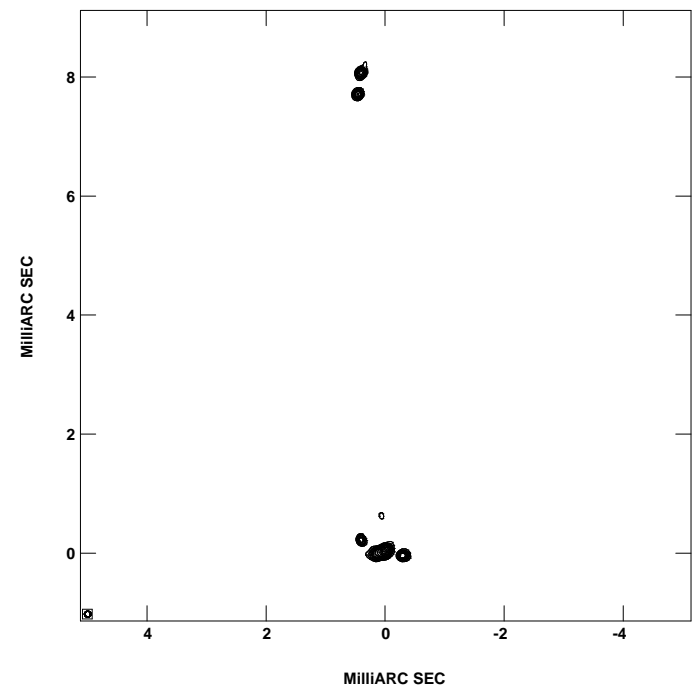

(c)

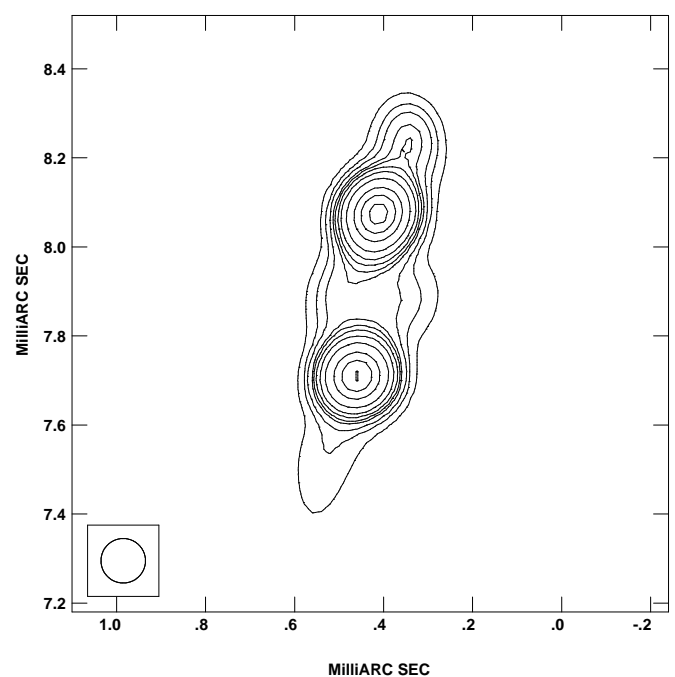

(b)

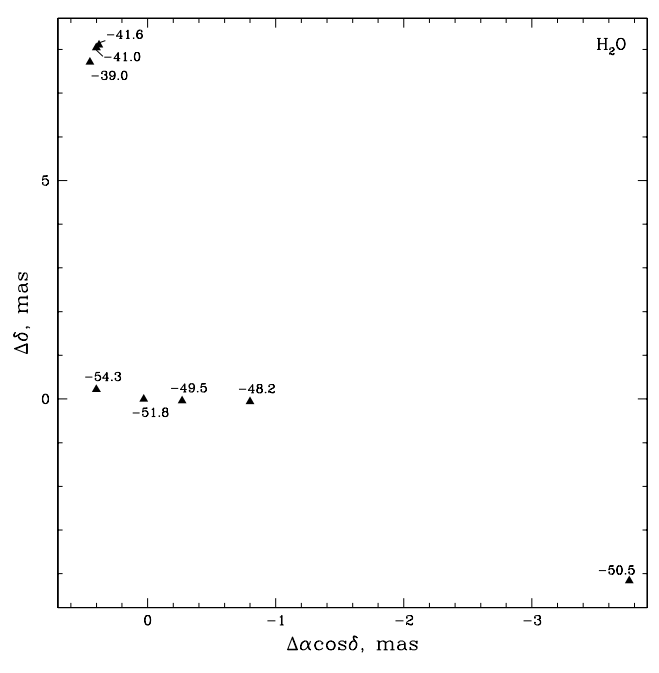

(d)

Figure 5: Summed maps of the $\mathrm{H}_{2} \mathrm{O}$ masers of the (a) southern and (b) northern groups. (c) Total map, where the brightness of each point is determined by the maximum of the brightnesses of the maps at that point for all spectral channels. The contours are drawn at 0.03, 0.05, 0.07, 0.1, 0.12, 0.15, $0.2,0.3,0.5,0.6,0.7,0.8$ and 0.9 of the maximum, which are $3.9 \mathrm{Jy} / \mathrm{beam}$ for map (a), $1.9 \mathrm{Jy} /$ beam for map (b), and $3.9 \mathrm{Jy} /$ beam for map (c). (d) Positions of the water maser spots in GL 2789 relative to the reference feature at $-51.8 \mathrm{~km} / \mathrm{s}$. 
The spots themselves are very compact, and are not resolved even on the longest VLBA baselines. Figure 6 shows the dependence of the correlated flux on the baseline length for one of the spots. We can see that the correlated flux is nearly independent of the baseline length, indicating that this maser spot is not resolved by any of the baselines. The remaining spots are also unresolved. The upper limit to their angular size is 0.05 mas (or $50 \mu \operatorname{arcsec}$ ), and the corresponding lower limit to their brigthness temperature is $2 \times 10^{13} \mathrm{~K}$.

3.3.2. The Methanol maser. The mapping of the methanol maser was carried out in the same way as for the $\mathrm{H}_{2} \mathrm{O}$ maser. The four spectral features visible in Fig. 1 correspond to four maser spots (Table 3), as we can see in Fig. 7a, which presents the image made from the 1998 data. The spots are located roughly along a north-south line and form two groups of two spots. The distance between the groups is about 100 mas, and the radial velocity of the southern group is $2.5 \mathrm{~km} / \mathrm{s}$ higher than that of the northern group. As for the $\mathrm{H}_{2} \mathrm{O}$ maser spots, the spots themselves are not resolved; their sizes are less than 2 mas, corresponding to a lower limit for the brightness temperature of $1.4 \times 10^{9} \mathrm{~K}$. Figure $7 \mathrm{~b}$ shows the map of the GL 2789 methanol maser emission obtained using the 2000 data. Since the methanol observations were carried out twice with a time interval of two years, we can estimate possible changes in the relative positions of the spots. A comparison of the two images indicates that the distances between the spots did not change significantly, by less than 2 mas, which is within the observational errors. The position

of spot $B$ changed by 2.5 mas in the line wings. The intensity and profile of the line corresponding to spot $B$ also varied. These variations may indicate a weakening of the previous spot $B$ and the appearance of another spot near the old position at a slightly different velocity.

\section{MODEL FOR THE MASERS}

Figure $7 \mathrm{c}$ shows the relative positions of the $\mathrm{H}_{2} \mathrm{O}$ and methanol masers. In both masers, the spots are distributed roughly along a north-south line, with the center of the methanol maser shifted 90 mas south from the center of the $\mathrm{H}_{2} \mathrm{O}$ maser. The accuracy of the position of the methanol maser relative to the water maser is not high, so that it is reasonable to suppose that both masers are located approximately on a north-south line similar to those corresponding to the structure of the maser spots. The maximum distance between the methanol-maser components is roughly a factor of ten 


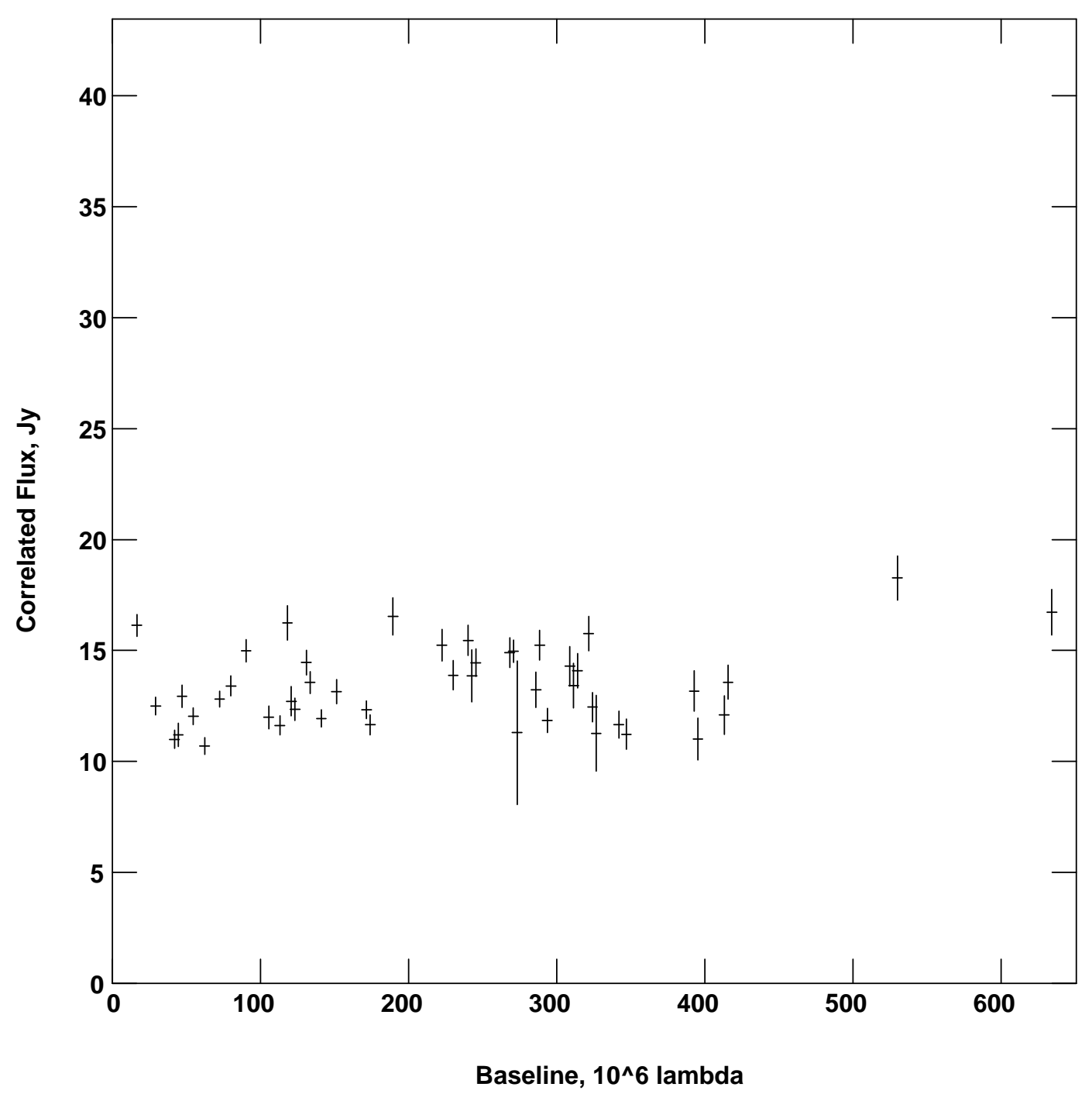

Figure 6: Dependence of the correlated flux on the baseline length for one of the water-maser spots (the feature at $-41.6 \mathrm{~km} / \mathrm{s}$ ).

greater than for the $\mathrm{H}_{2} \mathrm{O}$ maser, while the difference in radial velocities is about a factor of three smaller. The radial velocity of the molecular cloud associated with GL $2789,-45.6 \mathrm{~km} / \mathrm{s}$, is within the interval of velocities for the $\mathrm{H}_{2} \mathrm{O}$ maser and at the blue edge of the velocity interval for the methanol maser. The radial-velocity gradients for the methanol and $\mathrm{H}_{2} \mathrm{O}$ masers have different signs: while the methanol radial velocity decreases in the direction 
of the $\mathrm{H}_{2} \mathrm{O}$ maser, from south to north, the $\mathrm{H}_{2} \mathrm{O}$ radial velocity increases from south to north.

The variations of the radial velocity in the north-south direction are shown in Fig. 7d. These properties can be explained using a model in which the masers lie in a Keplerian disk viewed nearly edge-on rotating around the supposed central star. The position of the star, which may correspond to the optical object N0, is not known with sufficient accuracy to establish the parameters of the Keplerian orbit. As we can see from Table 1, both masers coincide with the object N0 to within the accuracy of its coordinates, 0 "'.2. From general considerations, it seems likely that the $\mathrm{H}_{2} \mathrm{O}$ masers are closer to the star than the methanol maser. This is supported by their large radialvelocity dispersion, consistent with the idea that the $\mathrm{H}_{2} \mathrm{O}$ masers move in Keplerian orbits at closer distances to the star, and the smaller linear scale of the $\mathrm{H}_{2} \mathrm{O}$ maser source, about $50 \mathrm{AU}$ (while the size of the region of methanolmaser emission is of the order of $600 \mathrm{AU})$. In addition, the $\mathrm{H}_{2} \mathrm{O}$ maser requires a higher gas temperature $(300-400 \mathrm{~K})$ and higher density $\left(\sim 10^{9} \mathrm{~cm}^{-3}\right)$, while the methanol masers require temperatures not exceeding 100 and gas densities not exceeding $10^{7} \mathrm{~cm}^{-3}$.

It is natural to suppose that the gas temperature and density are higher closer to the star than further from it. The opposite velocity gradients for the $\mathrm{H}_{2} \mathrm{O}$ and methanol masers are consistent with a disk model only if the star is located between the northern and southern components of the $\mathrm{H}_{2} \mathrm{O}$

Table 2: Relative positions of the $\mathrm{H}_{2} \mathrm{O}$ maser spots.

\begin{tabular}{|c|c|c|c|}
\hline $\begin{array}{l}\text { Radial } \\
\text { velocity, } \\
\mathrm{km} / \mathrm{s}\end{array}$ & $\begin{array}{l}\Delta \alpha \cos \delta \\
\operatorname{mas}\end{array}$ & $\begin{array}{l}\Delta \delta \\
\text { mas }\end{array}$ & Flux, Jy \\
\hline-54.3 & 0.40 & 0.22 & 4.7 \\
\hline-51.8 & 0.03 & 0.00 & 36.0 \\
\hline-50.5 & -3.76 & -4.16 & 1.8 \\
\hline-49.5 & -0.27 & -0.04 & 10.0 \\
\hline-48.2 & -0.80 & -0.06 & 0.6 \\
\hline-41.6 & 0.38 & 8.10 & 6.6 \\
\hline-41.0 & 0.40 & 8.04 & 6.4 \\
\hline-39.0 & 0.45 & 7.71 & 7.3 \\
\hline
\end{tabular}


Table 3: Relative positions of the $\mathrm{CH}_{3} \mathrm{OH}$ maser spots.

The positions are given relative to the reference water-maser spot at $-51.8 \mathrm{~km} / \mathrm{s}$. This shift was determined from the absolute-coordinate measurements and has an accuracy of about 50 mas. The accuracy of the relative coordinates of the spots for lines emitted by a single molecule is about 1 mas.

\begin{tabular}{|c|c|r|r|r|r|r|r|}
\hline \multicolumn{2}{|c|}{} & \multicolumn{3}{|c|}{1998 Observations } & \multicolumn{3}{c|}{2000 Observations } \\
\hline $\begin{array}{c}\text { Spectral } \\
\text { feature }\end{array}$ & $\begin{array}{c}\text { Radial } \\
\text { velocity } \\
\mathrm{km} \mathrm{s}^{-1}\end{array}$ & $\Delta \alpha \cos \delta$ & $\Delta \delta$ & Flux & $\Delta \alpha \cos \delta$ & $\Delta \delta$ & Flux \\
& -43.4 & 76.0 & -45.1 & 2.0 & 77.7 & -45.4 & 2.5 \\
$\mathrm{~A}$ & -42.5 & 85.0 & -65.0 & 2.5 & 83.5 & -64.4 & 1.2 \\
$\mathrm{~B}$ & -42.8 & 83.7 & -63.2 & 1.0 & 82.8 & -64.7 & 0.9 \\
$\mathrm{~B}_{1}^{*}$ & -40.8 & 82.9 & -134.2 & 2.0 & 82.8 & -134.5 & 1.7 \\
$\mathrm{C}$ & -40.4 & 94.2 & -143.2 & 2.2 & 94.1 & -143.3 & 3.0 \\
$\mathrm{D}$ & \multicolumn{7}{|c|}{} \\
\hline
\end{tabular}

* component corresponds to the wing of the feature at $-42.5 \mathrm{~km} / \mathrm{s}$.

maser. In that case, the opposite radial-velocity gradient for the $\mathrm{H}_{2} \mathrm{O}$ maser can be explained by the fact that the masers are distributed in the direction toward the star and have radial velocities that are close to the systematic radial velocity of the star. The methanol masers arise in directions tangential to the disk and have radial velocities equal to the velocity of rotation of the disk at the corresponding distance from the star. In GL 2789, we see only emission from the side of the disk that is moving away from the observer and experiences a red shift. Components arising in regions of the disk that are approaching and have blue shifts are not visible for some reason, possibly due to differences in the pumping conditions.

Figure 8 shows a schematic of the disk and the distribution of the masers and star. In this model, if the mass of the star is $10 M_{\odot}$, the $\mathrm{H}_{2} \mathrm{O}$ masers are located at a distance $R=41.1 \mathrm{AU}$, while the methanol masers are at distances between $200 \mathrm{AU}$ and $800 \mathrm{AU}$, for the adopted distance to the source of $6 \mathrm{kpc}$. This follows from the relation for a Keplerian disk [13]

$$
\frac{d V}{d x}=30 \mathrm{~km} \mathrm{~s}^{-1} \mathrm{AU}^{-1} \sqrt{M / R^{3}}
$$

where $\Delta V$ is the observed radial-velocity interval $(\Delta V=17.3 \mathrm{~km} / \mathrm{s}$ for the $\mathrm{H}_{2} \mathrm{O}$ maser), $\Delta$ is the apparent extent of the maser source $(\Delta=8$ mas (or 
$48 \mathrm{AU}), M$ is the mass of the star in solar masses, and $R$ is the distance from the star to the $\mathrm{H}_{2} \mathrm{O}$ maser in AU. This model is similar to the model for a disk associated with a black hole in the nucleus of the galaxy NGC 4258 [14]. In that case, $\mathrm{H}_{2} \mathrm{O}$ masers observed at radial velocities close to the systematic velocity of the galaxy are distributed near the direction toward the center of the disk, with high-velocity features located at tangential points of the disk. In our model, in contrast to the model for NGC 4258, the $\mathrm{H}_{2} \mathrm{O}$ masers are observed only in the direction toward the center of the disk, and the role of the high-velocity features is played by the methanol masers, which show only blue features.

The disk model for the $\mathrm{H}_{2} \mathrm{O}$ and methanol masers in GL 2789 can be verified if time variations in the radial velocities of the $\mathrm{H}_{2} \mathrm{O}$ components can be detected: in the model, the expected acceleration is $1.1 \mathrm{~km} / \mathrm{s}$ per year, and the proper motion of the $\mathrm{H}_{2} \mathrm{O}$ masers spots should be 0.52 mas/yr. Both effects are associated with rotation of the disk, whose linear velocity is $14.8 \mathrm{~km} / \mathrm{s}$ at a distance of $41.1 \mathrm{AU}$ from a star with a mass of $10 M_{\odot}$, where the $\mathrm{H}_{2} \mathrm{O}$ masers are located. The acceleration and proper motion of the methanol masers should be small, since they are observed in the tangential direction. The disk model predicts a Keplerian dependence of the radial velocity of the methanol masers on their distance from the star, $V \sim R^{-\frac{1}{2}}$. The results of observations shown in Fig. 7d are consistent with the Keplerian model, although the number of points is too small to draw definitive conclusions. No proper motions of the methanol maser spots relative to the reference feature were detected, as was noted above, and an upper limit to the proper motion of 2 mas over 2 yrs corresponds to an upper limit for the linear velocity of the relative motion of the maser spots of $28.5 \mathrm{~km} / \mathrm{s}$. This limit is consistent with the absence of proper motion during tangential motion of the maser spots.

We can also consider other models for the GL 2789 maser sources. Historically, the first models for $\mathrm{H}_{2} \mathrm{O}$ masers associated with massive stars were expanding-envelope models [15]. This type of model is also of interest for GL 2789, since it is known from optical observations that the star emits a stellar wind with a velocity of more than $600 \mathrm{~km} / \mathrm{s}$ [1]. However, such high velocities are not observed in the $\mathrm{H}_{2} \mathrm{O}$ and methanol maser lines. Torrelles et al. [16] suggest $\mathrm{H}_{2} \mathrm{O}$ masers as associated with one-sided jets ejected by the star. Such a jet is observed in the radio continuum of W75N [16]. However, no jets have been detected in the case of GL 2789; in addition, the radio continuum source is much larger than the maser-emission region [6], 


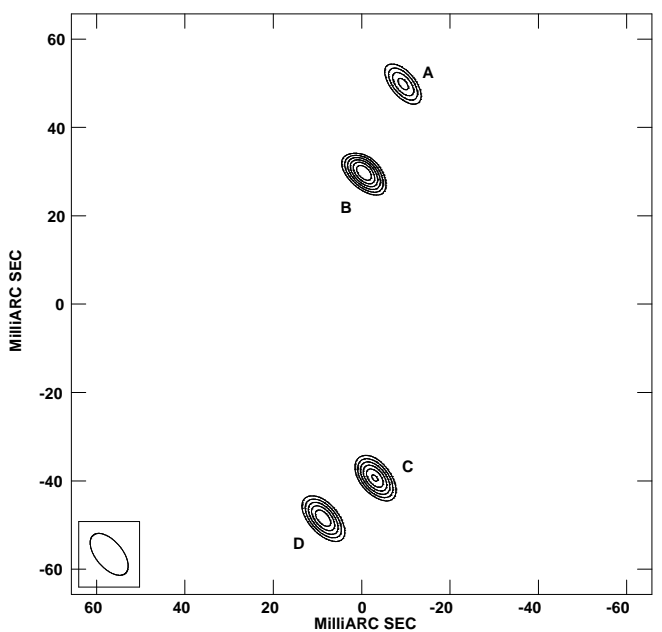

(a)

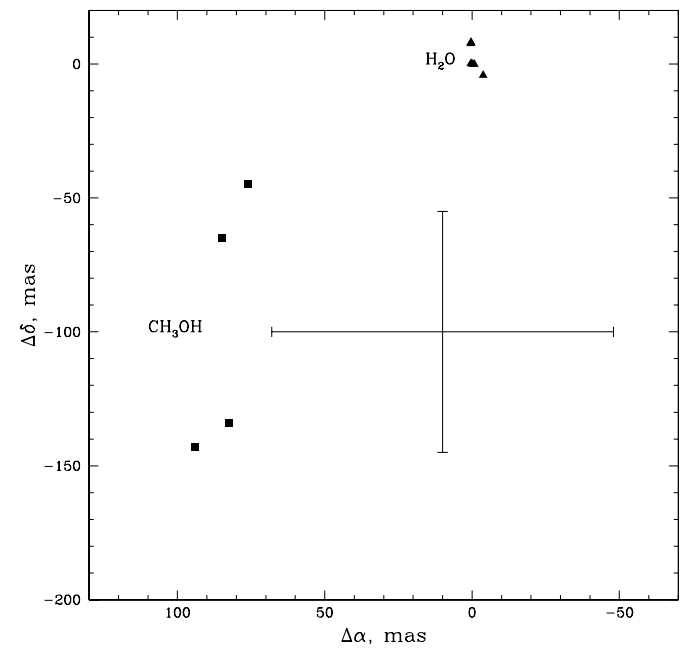

(c)

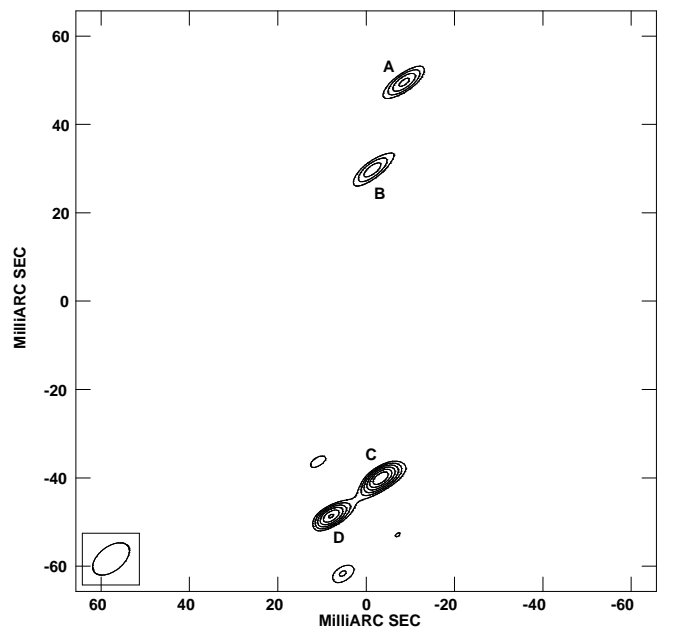

(b)

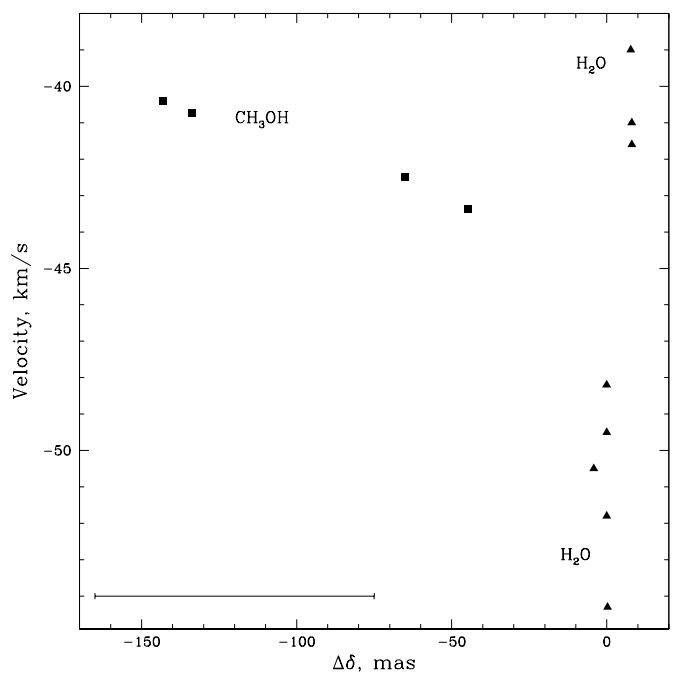

(d)

Figure 7: Map of the methanol maser in GL 2789. (a) 1998 observations with contours at $0.22 \times(4,5,6,7,8,9) \mathrm{Jy} /$ beam. (b) 2000 observations with contours at $0.35 \times(4,5,6,7,8,9) \mathrm{Jy} /$ beam. Components A, B, C, and D correspond to spectral features with velocities of $-43.4,-42.5,-40.7$, and -40.4 , respectively. (c) Relative positions of the methanol and water maser spots. The cross shows the error in the relative position of the methanol and water masers. (d) Variations of the radial velocity in the north-south direction. The error in the relative position of $\mathrm{H}_{2} \mathrm{O}$ and $\mathrm{CH}_{3} \mathrm{OH}$ masers is shown in the lower left corner. 
and is probably associated with the region of the stellar wind that is traced by the SII optical lines. The dimensions of the region of SII emission and of the region of radio-continuum emission coincide, as do the estimates of the mean density in this zone derived from these observations, of the order of $2 \times 10^{4} \mathrm{~cm}^{-3}$. The analysis of the SII line profile performed by Hamann and Persson [17] led them to conclude that, in addition to its stellar wind, V645 Cyg possesses a dense circumstellar disk. This was based on the presence of only blue components in the SII line profile, corresponding to material moving toward the observer. The red wing associated with the stellar-wind material moving away from the observer was absent from the SII spectrum, and Hamann and Persson [17] proposed that the emission in the red wing was screened by the disk. The presence of a disk is also supported by the emission in the KI, FeI, and CaII lines, which arise in comparatively cool material with a density of the order of $10^{10} \mathrm{~cm}^{-3}$.

Figure 6 of [17] shows a model with a disk surrounding the star, together with an extended region of stellar wind radiating in SII and $\mathrm{H}_{\alpha}$ lines. In this model, the $\mathrm{H}_{2} \mathrm{O}$ and methanol masers could be located in the disk proposed by Hamman and Persson [17]. Since the densities required for the $\mathrm{H}_{2} \mathrm{O}$, and especially the methanol, masers are lower than the density of the inner part of the disk derived from optical line data, these masers must be located in a lower-density region of the disk, at larger distances from the star.

In this model, the bipolar flow observed in GL 2789 [4] is emitted perpendicular to the disk and moves away from the star and masers at very large distances or tens of thousands of AU.

\section{DISCUSSION}

The detection of a class II methanol maser and the demonstration that it is associated with the optical object V645 Cyg, as well as the confirmation of the connection between this object and the $\mathrm{H}_{2} \mathrm{O}$ maser, provide additional arguments that V645 Cyg is a young stellar object. The source of thermal radio emission surrounding this object is most likely not a classical HII region, and instead a region filled with stellar wind.

Another important characteristic of this young stellar object is the presence of a cool circumstellar disk, in which compact sources of $\mathrm{H}_{2} \mathrm{O}$, methanol, and possibly $\mathrm{OH}$ maser emission are immersed. In [18], which was dedicated to the methanol masers in $\mathrm{W} 3(\mathrm{OH})$, it was suggested that individual features 


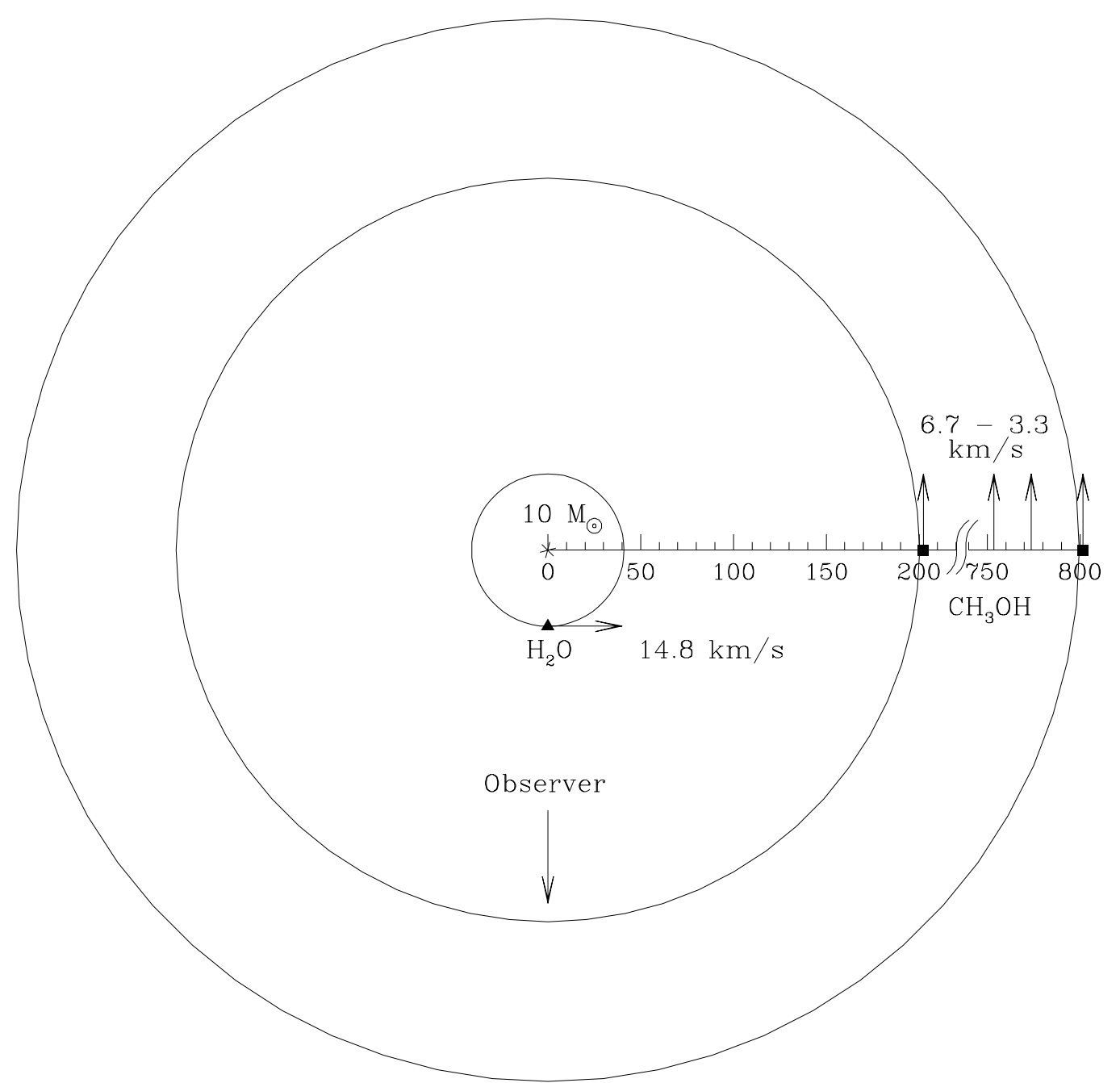

Figure 8: Schematic of the disk in GL 2789. The numbers show distance from the star in $\mathrm{AU}$, and the arrows show the direction and magnitude of the orbital velocity in $\mathrm{km} / \mathrm{s}$ for the $\mathrm{H}_{2} \mathrm{O}$ and methanol masers.

in the methanol and $\mathrm{OH}$ spectra were generated in extended envelopes surrounding ice planets. It is natural to suppose that such planets are located in a disk and move in Keplerian orbits. The chemical composition of these extended envelopes reflects the composition of the interstellar dust grains, or more precisely of their mantles. It is known that the mantles of dust grains consist primarily of ice, and methanol is the second most abundant 
ice material after water. For example, in the direction toward the high-mass protostars GL7009S and W33A, the abundances of methanol ice relative to water ice are 30\% and 5-22\% for GL7009S and W33A, respectively [19]. The methanol abundance in the direction of NGC 7538 IRS9 is 25\% [20], and is $8.7 \%$ in the direction of GL 2136 [21]. In other sources that have been studied, the methanol abundances does not exceed several per cent [19].

In cool disks surrounding protostars and very young stars, dust grains can adhere to larger bodies, ultimately reaching the dimensions of small planets. This is how the objects in Kuiper's belt were formed in the solar system. At sufficiently close distances from the star, water and methanol evaporate from the surfaces of planets and leave the gravitational fields of these planets if their masses are sufficiently low. Envelopes consisting primarily of water and methanol will thus form around these planets, whose extent can be much larger than the dimensions of a planet. This same picture is observed in miniature in our solar system, with cometary nuclei in place of ice planets. With approach toward the Sun, ice and methanol are evaporated from the surfaces of cometary nuclei, forming extended envelopes.

The water in such envelopes dissociates into atoms of hydrogen and molecules of $\mathrm{OH}$, and conditions favorable for water, $\mathrm{OH}$, and methanol maser emission are created: (a) rather high abundances, reaching $90 \%$ for water and $\mathrm{OH}$ and about $10 \%$ for methanol " - much higher than in the interstellar medium; (b) high column densities of water and methanol molecules "$10^{16} \mathrm{~cm}^{-2}$ for methanol and $10^{18} \mathrm{~cm}^{-2}$ for $\mathrm{H}_{2} \mathrm{O}$ for an envelope $10^{9} \mathrm{~cm}$ $(10000 \mathrm{~km})$ in size "- required to create high optical depths and, accordingly, high maser amplifications; (c) the required ranges of temperature and density "- from $400 \mathrm{~K}$ and $10^{9} \mathrm{~cm}^{-3}$ for the $\mathrm{H}_{2} \mathrm{O}$ masers to $100 \mathrm{~K}$ and $10^{6}-10^{7} \mathrm{~cm}^{-3}$ for the $\mathrm{OH}$ and class II methanol masers; (d) intense radiation near the rotational transitions of these molecules (submillimeter wavelengths and the far infrared), provided by reprocessing of the radiation of the young star by the dust.

These conditions are adopted in standard models for maser pumping. It is obvious that comets in the solar system are too small to form such extended dense envelopes; the optical depth in lines of $\mathrm{H}_{2} \mathrm{O}$ and methanol is too low, and no maser emission is observed. In GL 2789, the planets responsible for the water-vapor line emission move in Keplerian orbits at a distance from the star of about $40 \mathrm{AU}$, with each of the eight maser spots (Table 2) corresponding to a separate planet. The $\mathrm{OH}$ and methanol masers are located further from the star, at distances of 200-800 AU. At such large 
distances, the orbits of planets may not be located in a disk, due to possible perturbations in their motions. This could explain the presence of individual maser spots outside the disk.

This simple model can easily be verified using further observations. This requires monitoring of the radial velocities and positions of the maser spots to measure accelerations and linear translations due to the Keplerian motion. The most suitable masers for such observations are the $\mathrm{H}_{2} \mathrm{O}$ masers, which should be in the orbits that are closest to the star and move with the highest speeds. The corresponding estimates are presented in the previous

section. In the model presented here, the period of revolution of the planets responsible for the $\mathrm{H}_{2} \mathrm{O}$ maser emission is about 83 yrs. Therefore, using spectral and angular measurements with sufficiently high accuracy, it should be possible to determine the accelerations and velocities of the maser spots over fairly short time scales, less than one year. Such measurements must be conducted often due to the strong variability of the $\mathrm{H}_{2} \mathrm{O}$ maser emission. However, on short time scales, the accuracy of measurements such as propermotion measurements could prove to be insufficient, even using arrays with the longest possible baselines, such as the VLBA.

One possibility is the use of ground-space interferometers, which can attain angular resolutions exceeding that of ground-based interferometers by a factor of a few, or even an order of magnitude more than this. The measurement of proper motions using ground-space interferometers requires that the masers have sufficiently small angular sizes " - smaller than the width of the interferometer fringe. We have shown that the maser spots in GL 2789 are very compact, and are not resolved by the VLBA (Fig. 2b, Fig. 6). Therefore, they may be suitable for measurements using a ground-space interferometer. Other models with expanding components or jets predict that the spots should move uniformly and linearly, which could also be verified using observations with very high angular resolution.

\section{CONCLUSION}

The linear structure of the $\mathrm{H}_{2} \mathrm{O}$ and methanol masers is in best agreement with a disk model. If the mass of the star is $10 M_{\odot}$ and it is located at a distance of $6 \mathrm{kpc}$, the $\mathrm{H}_{2} \mathrm{O}$ maser components are located about $40 \mathrm{AU}$ from the star, with the methanol masers at distances of 200-800 AU. The physical objects responsible for the emission of individual maser spectral fea- 
tures could be ice planets surrounded by extended water-methanol envelopes moving in Keplerian orbits around the star. Such planets are similar to the objects of Kuiper's belt in the solar system. This model can be verified using observations with high angular resolution, using either ground-based or ground-space interferometers.

\section{ACKNOWLEDGEMENTS}

The authors affiliated with the Astro Space Center (VIS, MAV, and IEV) carried out this work in the framework of the RadioAstron project with partial support from the Russian Foundation for Basic Research (project code 01-02-16902), INTAS (grant 97-11451) and the State Centers for Science and Technology program "Astronomy" (grant 1.3.4.2). The observational data presented here were obtained using the VLBA of the National Radio Astronomy Observatory (USA), which is supported by the National Science Foundation and is operated under contract to Associated Universities, Inc., and also on the European VLBI Network, which is a consortium of European and Chinese radio astronomy institutes supported by the various national science councils.

\section{References}

[1] M. Cohen, Astrophys. J. 215, 533 (1977).

[2] R. M. Humphreys, K. M. Merrill, and J. H. Black, Astrophys. J. 237, L17 (1980).

[3] R. W. Goodrich, Astrophys. J. 311, 882 (1986).

[4] A. Schulz, J. H. Black, C. J. Lada, et al., Astrophys. J. 341, 288 (1989).

[5] J. M. Torrelles, G. Anglada, L. F. Rodríguez, et al., Astron. Astrophys. 177, 171 (1987).

[6] S. L. Skinner, A. Brown, and R. T. Stewart, Astrophys. J., Suppl. Ser. 87, 217 (1993).

[7] C. J. Lada, L. Blitz, M. J. Reid, and J. M. Moran, Astrophys. J. 243, 769 (1981). 
[8] G. Tofani, M. Felli, G. B. Taylor, and T. R. Hunter, Astron. Astrophys., Suppl. Ser. 112, 299 (1995).

[9] M. Morris and I. Kazés, Astron. Astrophys. 111, 239 (1982).

[10] V. I. Slysh, I. E. Val'tts, S. V. Kalenskii, et al., Astron. Astrophys., Suppl. Ser. 134, 115 (1999).

[11] V. Migenes, S. Horiuchi, V. I. Slysh, et al., Astrophys. J., Suppl. Ser. 123, 487 (1999).

[12] M. Szymczak, G. Hrynek, and A. J. Kus, Astron. Astrophys., Suppl. Ser. 143, 269 (2000).

[13] V. Minier, R. S. Conway, and R. S. Booth, Astron. Astrophys. 362, 1093 (2000).

[14] M. Miyoshi, J. Moran, J. Herrnstein, et al., Nature 373, 127 (1995).

[15] R. Genzel, D. Downes, J. M. Moran, et al., Astron. Astrophys. 66, 13 (1978).

[16] J. M. Torrelles, J. F. Gomez, L. F. Rodríguez, et al., Astrophys. J. 489, 744 (1997).

[17] F. Hamann and S. E. Persson, Astrophys. J. 339, 1078 (1989).

[18] V. I. Slysh, I. E. Val'tts, and S. V. Kalenskiŭ, et al., Astron. Zh. 76, 751 (1999) [Astron. Rep. 43, 657 (1999)].

[19] E. Dartois, W. Shutte, T. R. Geballe, et al., Astron. Astrophys. 342, L32 (1999).

[20] W. A. Shutte, A. G. G. M. Tielens, D. C. Whittet, et al., Astron. Astrophys. 315, L333 (1996).

[21] C. J. Skinner, A. G. G. M.Tielens, M. J. Barlow, and K. Justtanont, Astrophys. J. 399, L79 (1992).

Translated by D. Gabuzda 